\title{
EFFICACY AND SAFETY OF CARIPRAZINE IN ACUTE MANAGEMENT OF PSYCHIATRIC DISORDERS: A META-ANALYSIS OF RANDOMIZED CONTROLLED TRIALS
}

\author{
Hannah Cooper ${ }^{1}$, Raafat Mishriky ${ }^{2} \&$ Ayman Antoun Reyad ${ }^{3}$ \\ ${ }^{l}$ Midlands Partnership NHS Foundation Trust, Stafford, UK \\ ${ }^{2}$ Birmingham and Solihull Mental Health NHS Foundation Trust, Birmingham, UK \\ ${ }^{3}$ School of Pharmacy, Faculty of Science and Engineering, University of Wolverhampton, Wolverhampton, UK
}

received: 17.9.2019;

revised: 20.1.2020;

accepted: 28.1 .2020

\section{SUMMARY}

Background: Cariprazine is a new atypical antipsychotic drug approved for the treatment of schizophrenia and bipolar disorders. Methods: we searched the published randomized controlled-trials (RCT) to review cariprazine efficacy and tolerability using the databases (PubMed, EUDRACT, ClinicalTrials.gov and Cochrane Central Register of Controlled Trials) for cariprazine role in managing the following psychiatric conditions (schizophrenia, bipolar mania, bipolar depression and major depressive disorder). A meta-analysis was conducted using the identified 13 clinical trials to assess efficacy using with the outcomes: positive and negative syndrome scale (PANSS), clinical global impressions - severity of Illness (CGI-S), young mania rating scales (YMRS), Montgomery Asberg depression rating scale (MADRS) and Hamilton rating scale for depression (HAM-D). The risk of discontinuation due to adverse effects and common side effects were examined.

Results: The mean difference in change from baseline for PANSS was -6.23 (95\% Confidence Interval (CI) -7.18, -5.28) favoring cariprazine treatment $(p<0.00001)$. Similarly, mean difference for CGI-S was -0.36 (95\% CI -0.41, -0.30), YMRS -5.64 (95\% CI 6.86, -4.43), MADRS-1.43 (95\% CI -1.88, -0.99) and HAM-D -1.52 (95\% CI -2.28, -0.76). The risk ratio (RR) of discontinuing due to adverse events was 1.18 (95\% CI 1.01, 1.38) meaning risk increased by $18 \%$ in cariprazine group with RR for EPS related side effects 2.82 (95\% CI 2.47, 3.22) reflecting an increased risk of experiencing EPS related side effects by $182 \%$. Cariprazine was also associated with an increased incidence of side effects such as akathisia, nausea and insomnia.

Conclusion: Cariprazine demonstrates significant improvements in symptom intensity control in patients suffering from psychiatric conditions including schizophrenia, bipolar disorders and depression and is considered well-tolerated with similar rates of trials discontinuation; however, cariprazine was associated with a higher risk of EPS side effects. These findings will guide psychiatrists and pharmacists in their clinical role for supporting psychiatric patients care.

Key words: cariprazine - schizophrenia - bipolar disorders - major depressive disorder - psychiatric disorders

\section{INTRODUCTION}

Schizophrenia represent a major group of psychiatric disorders in which a person's perception, thoughts, mood and behavior are significantly altered (NICE 2014) and represents a collection of psychotic conditions that affects 21 million people worldwide, making it one of the top 10 causes of disability in developed countries (Schizophrenia Research Institute 2014). In England, psychotic disorders have an annual incidence of 32 cases per 100,000 people, 15 of these cases being schizophrenia (Kirkbride et al. 2012). A diagnosis of schizophrenia is confirmed by a psychiatrist after full psychiatric assessment using either the International Classification of Diseases (ICD-10) or the Diagnostic and Statistical Manual (DSM-5) criteria. The symptoms of schizophrenia can be divided into 'positive' symptoms, such as hallucinations and delusions, and 'negative' symptoms which affect the patients' ability to function such as lack of motivation (Rethink Mental Illness 2015). These Symptoms need to be present for a least one month before the diagnosis is made.

The causes of schizophrenia are not fully understood, however genetic factors could be involved (NICE
2014) with environmental factors including stress and traumatic life experiences, migration, urban living, cannabis use, complications before or during birth such as infections, poor nutrition (Andreasen 1999). A study exploring geographical variation effect on schizophrenia development showed that higher incidence in developing countries (Jablensky et al. 1992). 80\% of patients suffering with schizophrenia will have a relapse within 5 years of recovery (Robinson et al. 1999); this risk is substantially reduced by maintenance antipsychotic treatment (Leucht et al. 2012).

Antipsychotics first became available in the early 1950's allowing long-term stability for many people with schizophrenia (Schizophrenia Research Institute 2014) and in recent decades have been used in the treatment of bipolar disorders (Holder et al. 2017). There are two types of antipsychotics, typical (first generation) and atypical (second generation). First generation antipsychotics (FGA's) such as haloperidol and chlorpromazine are dopamine (D2) receptors antagonists and could block histamine, muscarinic and alpha-1 receptors (Ayano 2016). Second generation antipsychotics are serotonin-dopamine antagonists (Abi-Dargham \& Laruelle 2005). 5HT-2A antagonism can increase dopaminergic neurotransmission 
in the nigrostriatal pathway, which reduces the risk of extrapyramidal symptoms such as dystonic reactions, akathisia and tardive dyskinesia (Correll et al. 2004). Second generation antipsychotics (SGA) main side effects include weight gain, glucose intolerance and hyperprolactinemia (Ndukwe \& Nishtala 2017, Sapra et al. 2016).

Cariprazine is a new atypical antipsychotic drug approved by the FDA in 2015 for the treatment of schizophrenia and bipolar disorder (Caraci et al. 2017). Cariprazine acts as a D2 and D3 partial agonist with a special higher potency for the D3 receptor than dopamine (Stahl 2016); which differs from current antipsychotics whose main targets are D2 and 5-HT2A receptors (Abi-Dargham \& Laruelle 2005). The metabolism of cariprazine is via the CYP3A4 and CYP2D6 pathways. Two clinically relevant metabolites desmethyl-cariprazine, and didesmethyl-cariprazine have similar pharmacological activity to cariprazine although didesmethyl-cariprazine has a much longer half-life (1-3 weeks), compared to cariprazine (2-4 days) (Nakamura et al. 2016, Earley et al. 2017). Cariprazine common side effects include restlessness, akathisia and insomnia (Stahl 2016, Nakamura et al. 2016). In this systematic review/meta-analysis, we investigate the efficacy, tolerability and safety of cariprazine in adult patients ( $\geq 18$ years) suffering from different psychiatric conditions such as schizophrenia, bipolar disorders and depression using published randomized controlled-trials (RCT). To our knowledge, this is the first meta-analysis to cover role of cariprazine in managing depressive symptoms while updating our knowledge with latest findings in the field of schizophrenia and mania.

\section{METHODS}

\section{Study population and search strategy}

The study population includes adult patients (18-65 years old) taking part in phase II/III RCT's assigned to either the cariprazine $1.5-12 \mathrm{mg} /$ day, or placebo for the management of schizophrenia, bipolar mania, bipolar depression and major depressive disorder. A literature search was performed to find RCT's investigating the efficacy and/or safety of cariprazine compared to placebo. The search terms 'cariprazine' was used to search PubMed, EUDRACT, ClinicalTrials.gov and Cochrane Central Register of Controlled Trials. No restrictions on study size, year of study or duration were set. Titles were screened for relevance and duplicates were removed. Abstracts were then screened before the remaining relevant full texts were screened to see if they met the inclusion criteria (Figure 1).

\section{Inclusion and Exclusion Criteria}

Published phase II and III randomized controlled trials that investigate the tolerability, safety or efficacy of cariprazine in patients suffering from schizophrenia, bipolar mania, bipolar depression and major depressive disorder were included (Table 1). All RCT's were placebo controlled and doubled blinded to reduce the risk of bias.

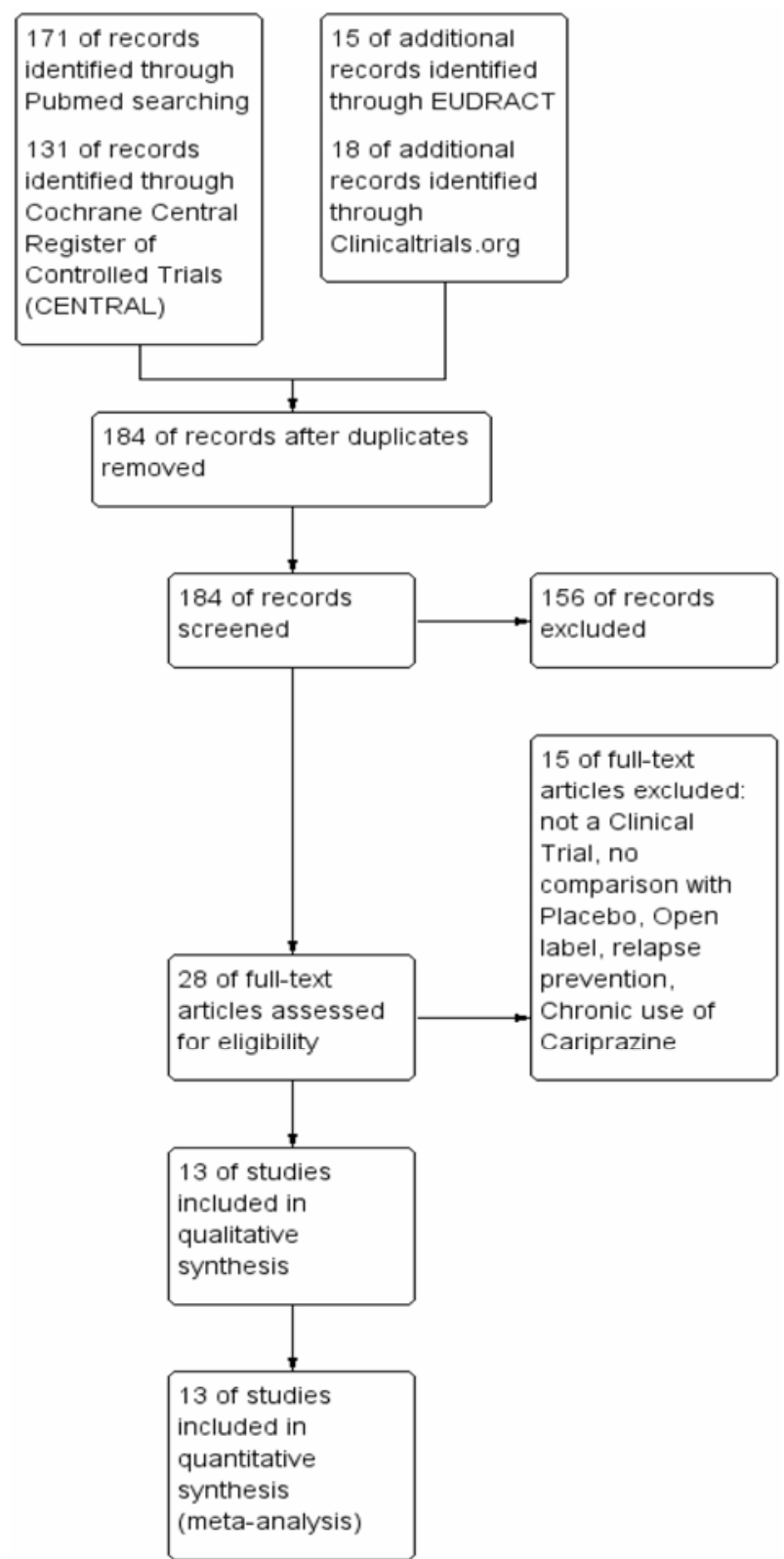

Figure 1. Flowchart summarizing the studies selection process

\section{Outcome measures}

The primary efficacy outcomes of cariprazine were Positive and Negative Syndrome Scale (PANSS), Clinical Global Impressions-Severity of Illness Score (CGIS), Young Mania Rating Scale (YMRS), Montgomery Asberg depression rating scale (MADRS) and Hamilton rating scale for depression (HAM-D) with mean changes from baseline recorded. Cariprazine treatment groups were combined $(1.5-12 \mathrm{mg} / \mathrm{d})$ and compared with placebo, while cariprazine doses outside this range were excluded. The thirteen RCTs (Calabrese et al. 2015, Durgam et al. 2014, 2015a,b, 2016a,b,c, Kane et al. 2015, Sachs et al. 2015, Earley et al. 2018, Fava et al. 2018, NCT02670538 2019, NCT02670551 2019) had a total of 3475 patients treated with cariprazine compared to 2086 patients who received placebo. All the 
trials included in the meta-analysis assessed CGI-S, seven RCTs assessed PANSS, 3 assessed YMRS, 9 assessed MADRS, while just 3 contained HAM-D (Figure 2).

The primary tolerability and safety outcomes for cariprazine were discontinuation due to adverse effects and EPS-related side effects. EPS-related side effects included akathisia, extrapyramidal disorder, restlessness and tremor.

\section{Statistical Analysis}

Review Manager 5.3 (RevMan) along with the Cochrane Collaboration tool for assessing the risk of bias (Higgins et al. 2011, The Nordic Cochrane Centre, The Cochrane Collaboration 2014) were used to assess the levels of selection, performance, detection, attrition and reporting bias in each of the 13 RCT's. 'Characteristics of study' tables were completed in RevMan for each of the individual studies and a summary table was created (The Nordic Cochrane Centre, The Cochrane Collaboration 2014). Funnels plots for each of the outcomes were also created (Guyatt et al. 2011, Sterne et al. 2011).

The inverse variance method with random effects model was used to calculate the mean differences for continuous outcomes (PANSS, CGI-S, YMRS, MADRS and HAM-D). The Mantel-Haenszel method with random effects model was used to calculate the risk ratio for all dichotomous outcomes (Risk of discontinuation due to adverse effects, common side effects and EPS related side effects) (Egger et al. 2001). RevMan was used for all statistical analysis, $95 \%$ confidence intervals were used for all outcomes and p-value $<0.05$ was regarded as statistically significant (The Nordic Cochrane Centre, The Cochrane Collaboration 2014).

Table 1. Randomized Controlled trials included in the meta-analysis

\begin{tabular}{|c|c|c|c|c|c|}
\hline Study & Design & Indication & Duration (weeks) & Dose range $(\mathrm{mg} / \mathrm{d})$ & Population \\
\hline Calabrese 2015 & $\begin{array}{l}\text { Double blinded, } \\
\text { Placebo control }\end{array}$ & Bipolar I mania & 3 & $\begin{array}{l}\text { Placebo } \\
\text { Car 3-6 } \\
\text { Car 6-12 }\end{array}$ & $\begin{array}{l}161 \\
167 \\
169\end{array}$ \\
\hline Durgam 2015b & $\begin{array}{l}\text { Double blinded, } \\
\text { Placebo control }\end{array}$ & Bipolar I mania & 3 & $\begin{array}{l}\text { Placebo } \\
\text { Car 3-12 }\end{array}$ & $\begin{array}{l}118 \\
118\end{array}$ \\
\hline Sachs 2015 & $\begin{array}{l}\text { Double blinded, } \\
\text { Placebo control }\end{array}$ & Bipolar I mania & 3 & $\begin{array}{l}\text { Placebo } \\
\text { Car 3-12 }\end{array}$ & $\begin{array}{l}158 \\
154\end{array}$ \\
\hline Durgam 2014 & $\begin{array}{l}\text { Double blinded, } \\
\text { Placebo control }\end{array}$ & Schizophrenia & 6 & $\begin{array}{c}\text { Placebo } \\
\text { Car } 1.5 \\
\text { Car } 3 \\
\text { Car } 4.5\end{array}$ & $\begin{array}{l}151 \\
145 \\
146 \\
147\end{array}$ \\
\hline Durgam 2015 & $\begin{array}{l}\text { Double blinded, } \\
\text { Placebo control }\end{array}$ & Schizophrenia & 6 & $\begin{array}{c}\text { Placebo } \\
\text { Car } 3 \\
\text { Car } 6\end{array}$ & $\begin{array}{l}153 \\
155 \\
157\end{array}$ \\
\hline Durgam 2016 & $\begin{array}{l}\text { Double blinded, } \\
\text { Placebo control }\end{array}$ & Schizophrenia & 6 & $\begin{array}{c}\text { Placebo } \\
\text { Car 1.5-4.5 } \\
\text { Car 6-12 }\end{array}$ & $\begin{array}{l}129 \\
127 \\
133\end{array}$ \\
\hline Kane 2015 & $\begin{array}{l}\text { Double blinded, } \\
\text { Placebo control }\end{array}$ & Schizophrenia & 6 & $\begin{array}{l}\text { Placebo } \\
\text { Car 3-6 } \\
\text { Car 6-9 }\end{array}$ & $\begin{array}{l}147 \\
151 \\
148\end{array}$ \\
\hline Durgam 2016c & $\begin{array}{l}\text { Double blinded, } \\
\text { Placebo control }\end{array}$ & $\begin{array}{l}\text { Bipolar } \\
\text { Depression }\end{array}$ & 8 & $\begin{array}{l}\text { Placebo } \\
\text { Car } 1.5 \\
\text { Car } 3\end{array}$ & $\begin{array}{l}141 \\
145 \\
145\end{array}$ \\
\hline Earley 2019 & $\begin{array}{l}\text { Double blinded, } \\
\text { Placebo control }\end{array}$ & $\begin{array}{l}\text { Bipolar } \\
\text { Depression }\end{array}$ & 6 & $\begin{array}{l}\text { Placebo } \\
\text { Car } 1.5 \\
\text { Car } 3\end{array}$ & $\begin{array}{l}163 \\
160 \\
165\end{array}$ \\
\hline NCT02670538 & $\begin{array}{l}\text { Double blinded, } \\
\text { Placebo control }\end{array}$ & $\begin{array}{l}\text { Bipolar } \\
\text { Depression }\end{array}$ & 6 & $\begin{array}{c}\text { Placebo } \\
\text { Car } 1.5 \\
\text { Car } 3\end{array}$ & $\begin{array}{l}165 \\
167 \\
158\end{array}$ \\
\hline Earley 2018 & $\begin{array}{l}\text { Double blinded, } \\
\text { Placebo control }\end{array}$ & $\begin{array}{l}\text { Major Depressive } \\
\text { Disorder }\end{array}$ & 18 & $\begin{array}{c}\text { Placebo } \\
\text { Car 1.5-4.5 }\end{array}$ & $\begin{array}{l}261 \\
269\end{array}$ \\
\hline Durgam 2016b & $\begin{array}{l}\text { Double blinded, } \\
\text { Placebo control }\end{array}$ & $\begin{array}{l}\text { Major Depressive } \\
\text { Disorder }\end{array}$ & 8 & $\begin{array}{l}\text { Placebo } \\
\text { Car 2-4.5 }\end{array}$ & $\begin{array}{l}269 \\
276\end{array}$ \\
\hline Fava 2018 & $\begin{array}{l}\text { Double blinded, } \\
\text { Placebo control }\end{array}$ & $\begin{array}{l}\text { Major Depressive } \\
\text { Disorder }\end{array}$ & 19 & $\begin{array}{l}\text { Placebo } \\
\text { Car 1-2 }\end{array}$ & $\begin{array}{l}81 \\
73\end{array}$ \\
\hline
\end{tabular}



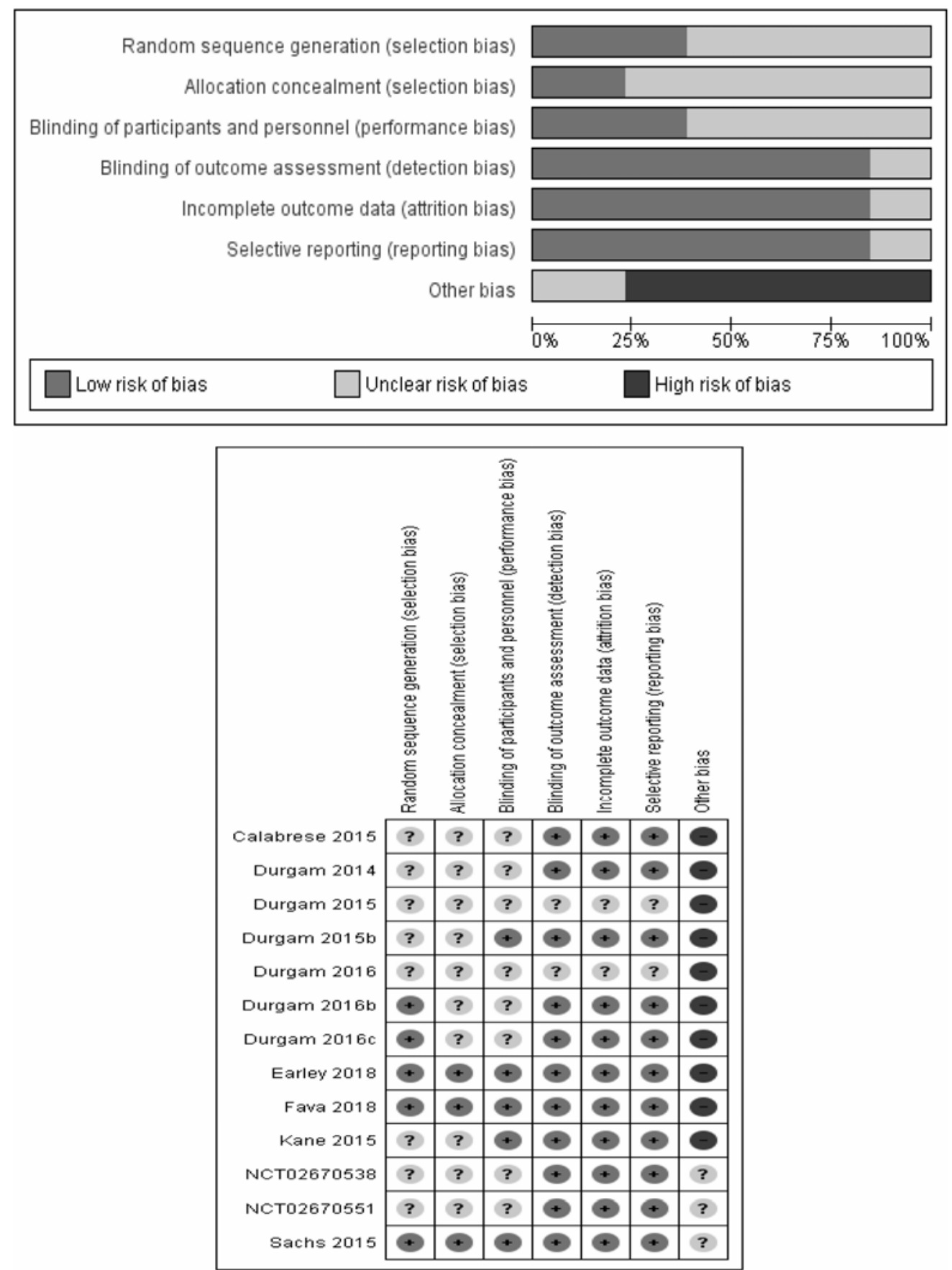

Figure 2. Risk of Bias of the $13 \mathrm{RCT}$ included in the meta-analysis

\section{RESULTS}

\section{Search results and included studies}

Figure 1 shows the selection process of RCT's included. PubMed, EUDRACT, ClinicalTrials.gov and Cochrane Central Register of Controlled Trials were searched for 'cariprazine' giving 335 records in total. After removing duplicates and screening titles and abstracts, 28 studies were included in full text screening, while 13 RCTs met the inclusion criteria (Calabrese et al. 2015, Durgam et al. 2014, 2015a,b, 2016a,b,c, Kane et al. 2015, Sachs et al. 2015, Earley et al. 2018, Fava et al. 2018, NCT02670538 2019, NCT02670551 2019).
Table 1 shows the characteristics of the included RCTs. All of them were double blinded and placebo controlled. Treatment duration ranged from 3 to 18 weeks and cariprazine dose ranged from 1.5 to $12 \mathrm{mg} /$ day. Any active controlled groups as well as doses of cariprazine out of the inclusion range of 1.5$12 \mathrm{mg} / \mathrm{d}$ were ignored. Four studies investigated cariprazine use in schizophrenia, three in bipolar mania, 3 in bipolar depression and 3 in major depressive disorder (Table 1). Studies were undertaken in regions including USA, Russia, Ukraine and India with similar prevalence and incidence rates to the UK (Steel et al. 2014). 

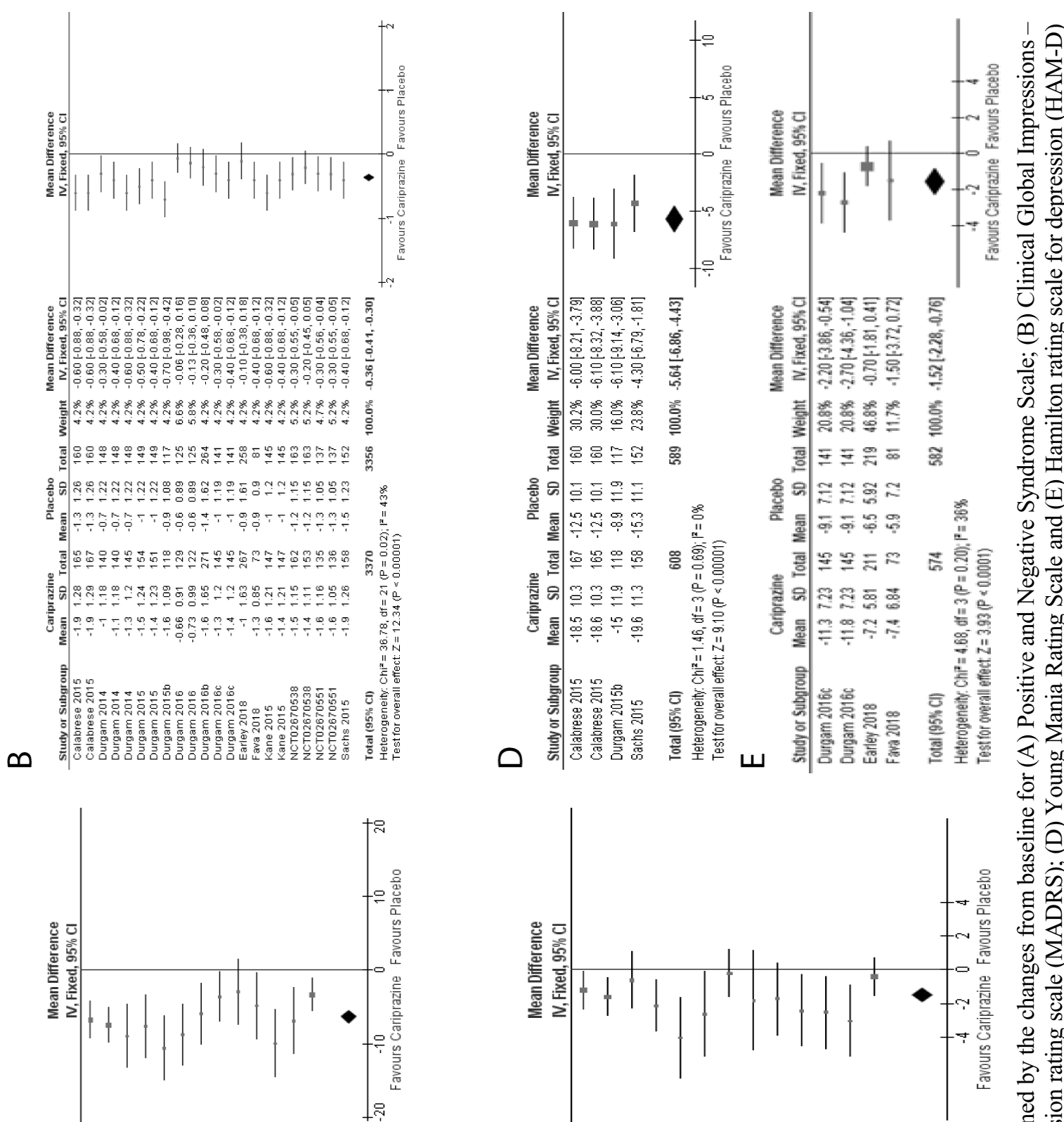

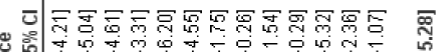

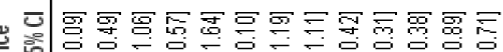

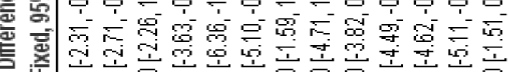

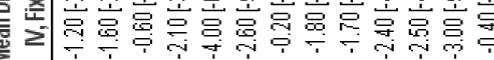

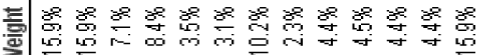

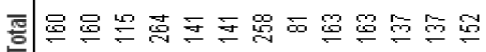

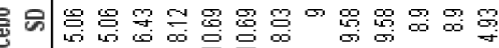

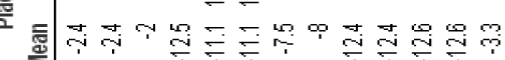

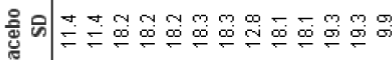

总

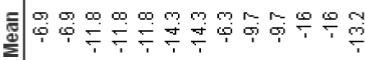

鱼言

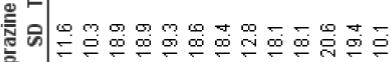

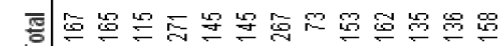

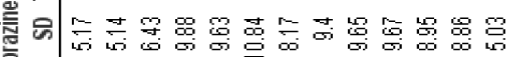

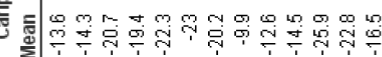

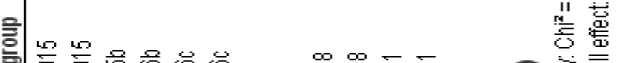




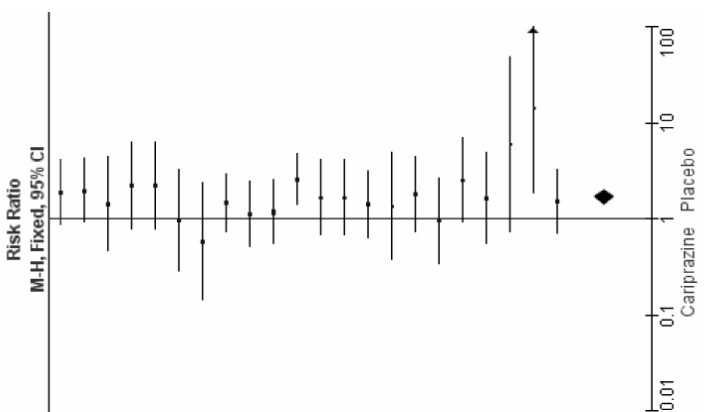

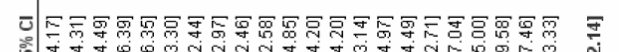

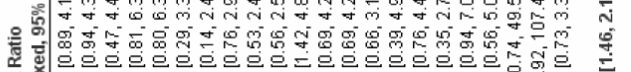

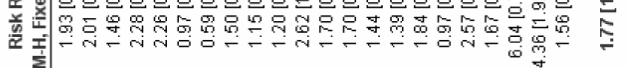

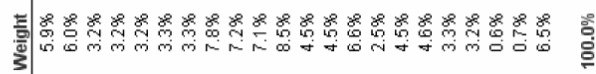

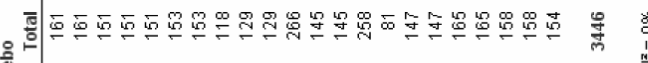

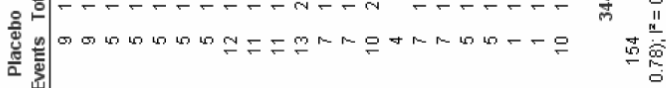

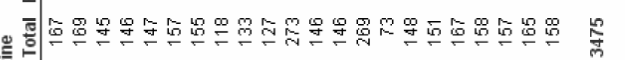
窟高 咅

要

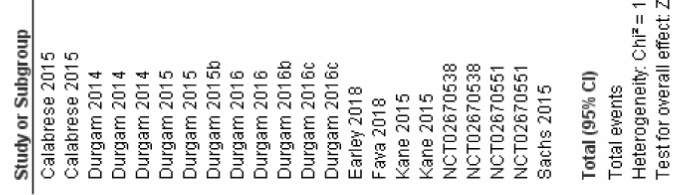

$\infty$

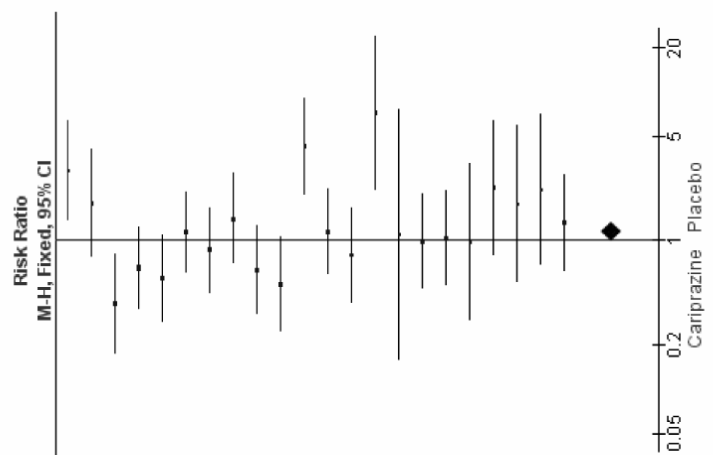

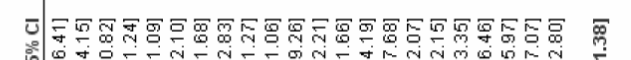

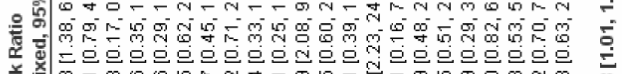

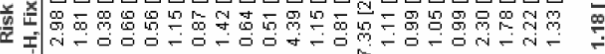
돌

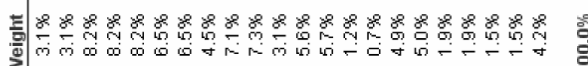

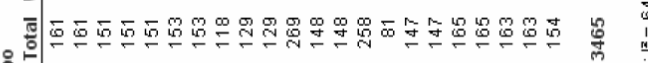

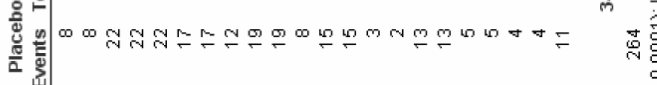

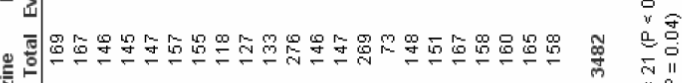

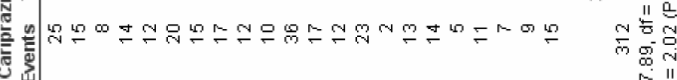

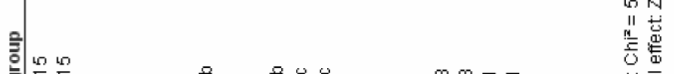

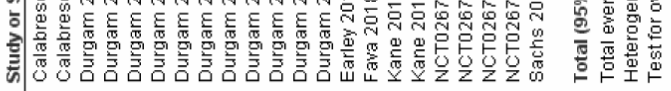
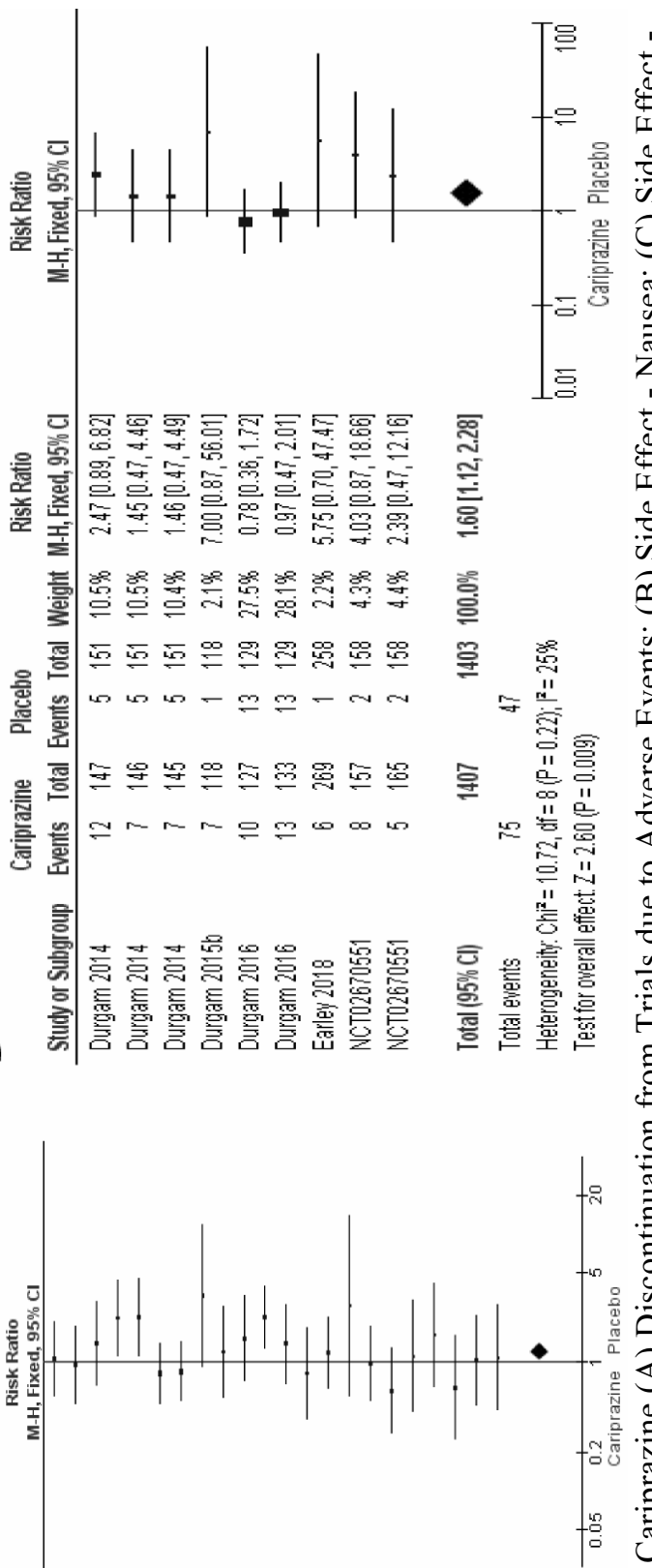

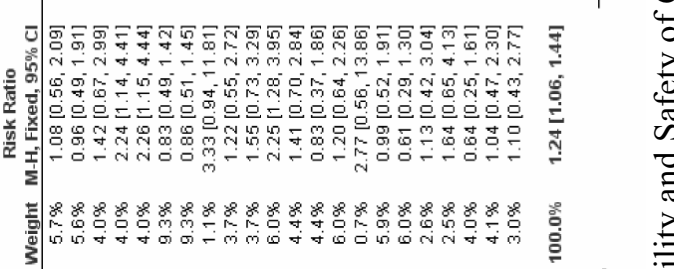

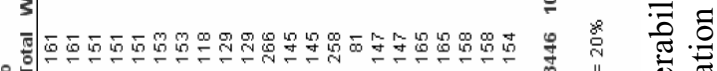

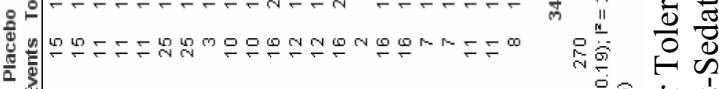

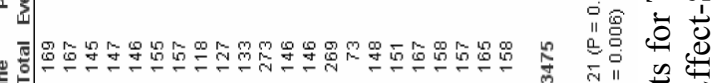
总

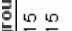

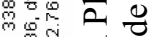

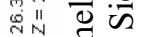

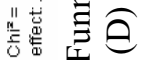
U 


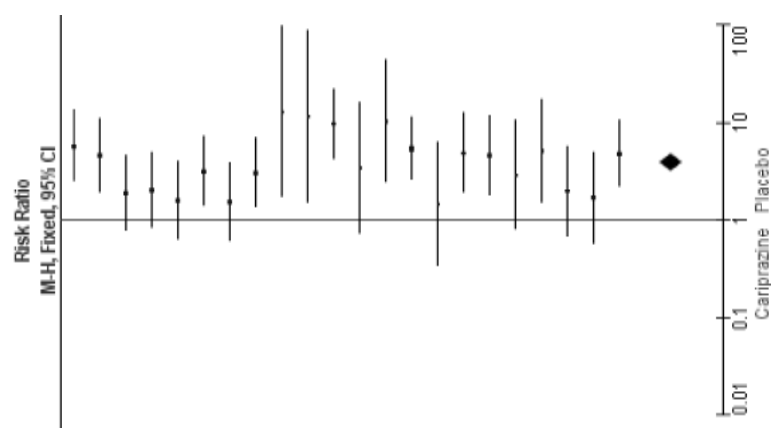

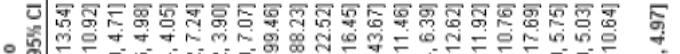

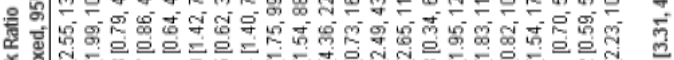

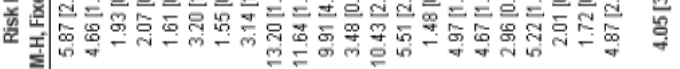

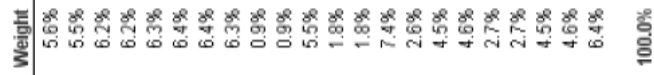

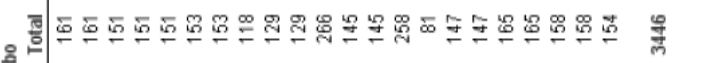

熝

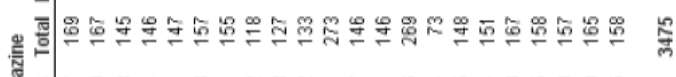

空

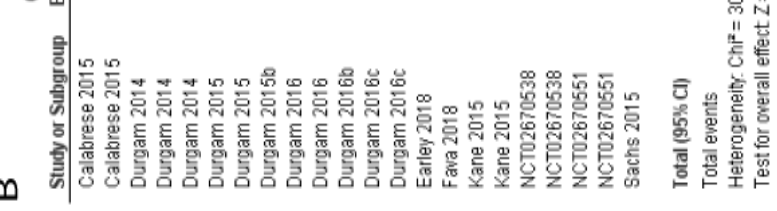

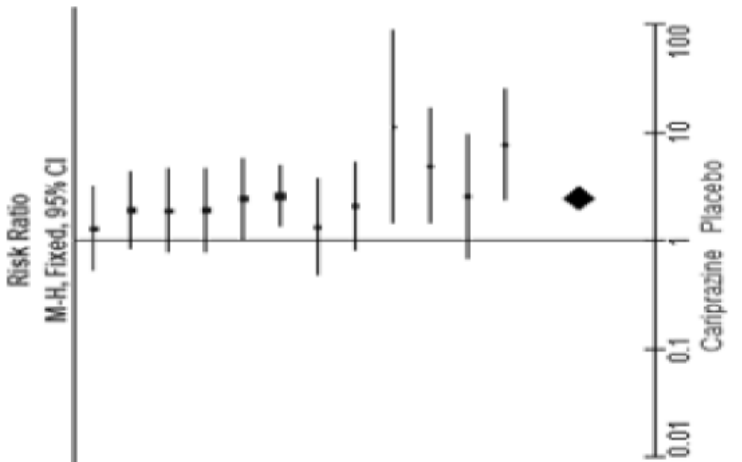

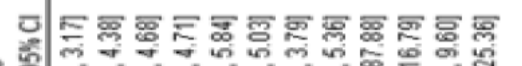

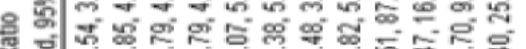

할 준

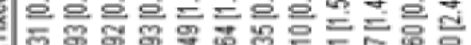

辤

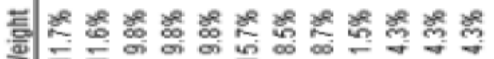

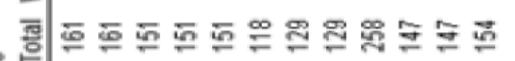

总

言

들

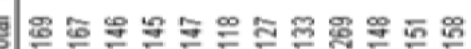

突

$=\infty m m=9 m \div \div$

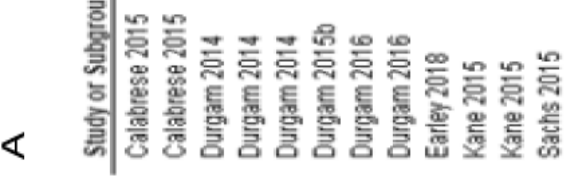

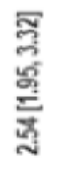

홍

ळ

$\therefore$ 商

क

롤

$\stackrel{\square}{=}$

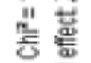

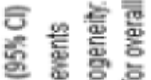

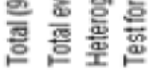

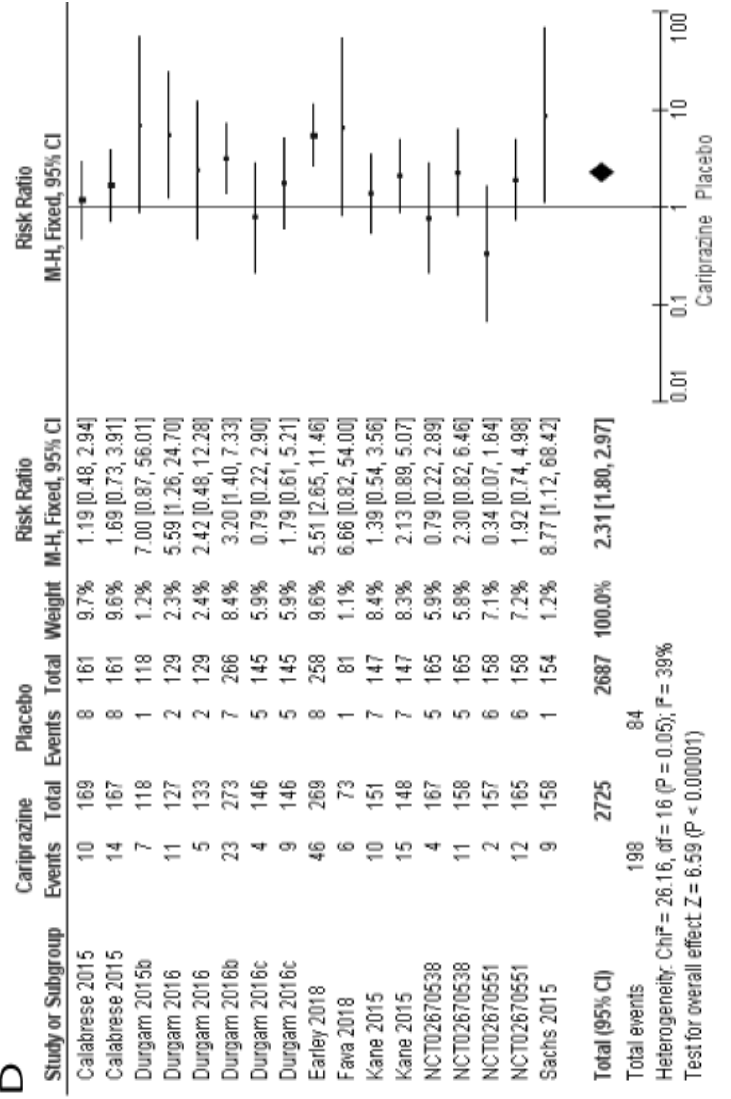

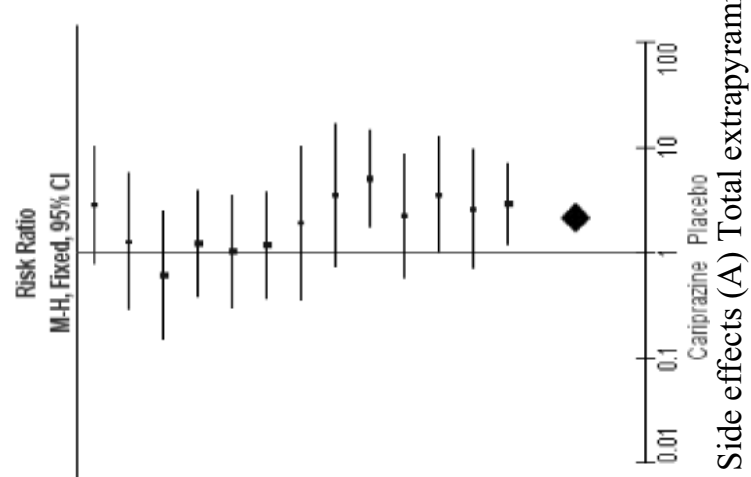

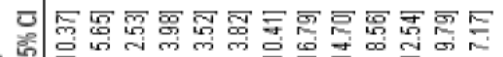

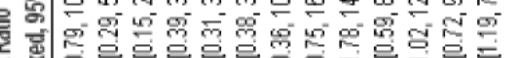

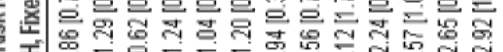

폴

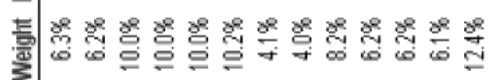

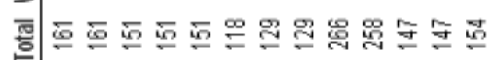

黄

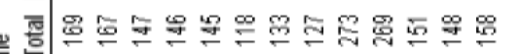

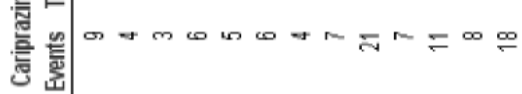

్ㅗㄹ

흥

ำ

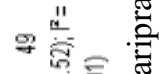

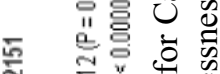

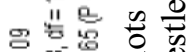

戛

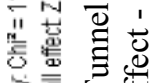

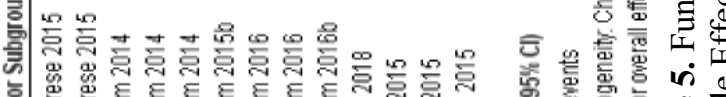

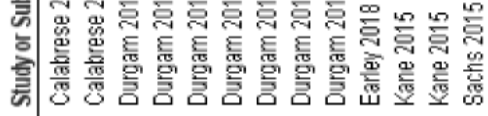


Figure 2 shows the risk of bias risk. The sequence generation, allocation concealment and blinding were mostly with 'unclear' risk due to insufficient information. The patients in all the studies were randomly assigned and there was certain level of blinding for both participants and personnel. The domains relating to the completeness of data and reporting of outcomes were 'low' risk of bias, while other risks was high due to the high extent of support of trials by pharmaceutical companies (Figure 2).

\section{Efficacy of Cariprazine in Psychiatric disorders}

The mean change from baseline in PANSS total score was significantly greater for cariprazine compared to placebo, with a mean difference of -6.23 (95\% Confidence Interval (CI) -7.18, -5.28) favoring cariprazine treatment $(p<0.00001)$ (Figure 3a). The forest plot shows the mean differences for all the studies individually favour cariprazine, with some degree of heterogeneity between the studies $\left(\chi^{2}=36.78, \mathrm{p}=0.03, \mathrm{I}^{2}=43 \%\right)$.

The mean change from baseline in CGI-S was significantly greater for cariprazine with a mean difference of -0.36 (95\% CI $-0.41,-0.30), \mathrm{p}<0.00001$, which was clinically and statistically significant (Figure $3 \mathrm{~b}$ ). The forest plot shows that all the studies individually favour the cariprazine treatment, with low to moderate heterogeneity between the studies $\left(\mathrm{I}^{2}=43 \%\right)$.

The mean change from baseline in MADRS score was significantly greater for cariprazine compared to placebo, with a mean difference of -1.43 (95\% CI -1.88 , $-0.99)$ favoring cariprazine treatment $(\mathrm{p}<0.00001)$ (Figure $3 \mathrm{~d}$ ). All the studies individually favour cariprazine, with some degree of heterogeneity between the studies $\left(\chi^{2}=17.75, \mathrm{I}^{2}=32 \%\right)$.

Three RCTs were used to measure the effect of cariprazine on Young Mania Rating Scale (Figure 3c). The overall result showed cariprazine positive effect on YMRS score with mean difference -5.64 (95\% CI -6.86, $-4.43)$, with very low heterogeneity among the studies $\left(\mathrm{I}^{2}=0 \%\right)$ as these studies showed similar outcome, chisquare was $1.46, \mathrm{p}=0.51$ showing homogeneity among the studies.

The mean change from baseline in HAM-D score was significantly greater for cariprazine compared to placebo, with a mean difference of $-1.52(95 \%$ CI -2.28 , $-0.76)$ favoring cariprazine treatment $(p<0.00001)$ with all the studies individually favour cariprazine, heterogeneity $\left(\chi^{2}=4.68, \mathrm{I}^{2}=36 \%\right)$.

\section{Tolerability and Safety of Cariprazine in Psychiatric disorders}

The overall risk ratio for trial discontinuation due to adverse effects is 1.18 (1.01 to 1.38 ), $\mathrm{p}=0.75$, presenting an increase by $18 \%$ in the cariprazine group (Figure $4 a$ ). There is variation among the studies with some favoring cariprazine, while others favoring placebo; with a moderate to high heterogeneity $\left(\mathrm{I}^{2}=64 \%\right)$.
Cariprazine was associated with some side effects including nausea $R R=1.77$ (1.46 to 2.14), $\mathrm{p}<0.00001$; insomnia $R R=1.24$ (1.06 to 1.44$), p=0.006$ and sedation $\mathrm{RR}=1.60$ (1.12 to 2.28), $\mathrm{p}=0.009$ (Figure 4).

Akathisia, Extrapyramidal Disorder, Restlessness and Tremor were examined individually (Figure 5). The risk ratio of Akathisia was 4.05 (95\% CI, 3.31 to 4.97 ) with $13 \%$ of patients in cariprazine group experiencing akathisia compared to just $3.2 \%$ in the placebo group. Extrapyramidal disorder had a risk ratio of 2.54 (95\% CI, 1.95 to 3.32 ) with $9.5 \%$ of patient in the cariprazine group experiencing this side effect compared to just $3.8 \%$ in the placebo group. $7.3 \%$ experienced restlessness in cariprazine group compared to just $3.1 \%$ in the placebo group, while $5.1 \%$ experiencing tremor in the cariprazine group compared to $2.3 \%$ in the placebo group.

\section{DISCUSSION}

This systematic review/meta-analysis investigated the efficacy and safety of cariprazine for the management of psychiatric conditions including schizophrenia, bipolar disorders and major depressive disorder using the available clinical trials. Cariprazine improved PANSS total- psychiatric scale used for measuring symptom severity in patients with psychosis along with improving anxiety and depressed mood. Cariprazine also showed significant improvements in CGI-S, YMRS, MADRS and HAM-D. As far as our awareness, this is the first meta-analysis that covered the role of cariprazine in the different psychiatric conditions, our results are in consistency with a previous meta-analysis that focused on the tolerability of cariprazine and showed statistically significant higher risk of EPS-related AEs (Lao et al. 2016). These results confirm similar results in another meta-analysis that studied cariprazine in psychotic disorders such as schizophrenia, but not including recent trials especially in the field of cariprazine role in the management of bipolar depression and major depressive disorder (Chhatlani et al. 2018). Risk of discontinuing was similar in cariprazine compared to placebo with increased risk of EPS in the cariprazine treatment group, especially akathisia.

This review shows that cariprazine is well tolerated and significantly improves schizophrenia, bipolar disorder and major depression management. However, the results need to be interpreted with caution as the treatment length ranged from just 3 to 18 weeks, with no enough evidence for long-term treatment effects, while several doses of cariprazine were used with different efficacy and side effects profile. Therefore, it is recommended that further research using different doses with long-treatment is conducted for a more comprehensive understanding of cariprazine role in the management of psychiatric conditions. 


\section{CONCLUSIONS}

Cariprazine demonstrates significant improvements in symptom intensity control in patients suffering from psychiatric conditions including schizophrenia, bipolar disorders and depression and is considered well-tolerated with similar rates of trials discontinuation; however, cariprazine was associated with a higher risk of EPS side effects. These findings will guide psychiatrists and pharmacists in their clinical role for supporting psychiatric patients care.

\section{Acknowledgements: None.}

Conflict of interest: None to declare.

\section{Contribution of individual authors:}

Hannah Cooper \& Ayman Antoun Reyad: literature searches and analysis, statistical analysis, manuscript writing.

Raafat Mishriky: interpretation of data, manuscript writing.

\section{References}

1. Abi-Dargham A \& Laruelle M: Mechanisms of action of second generation antipsychotic drugs in schizophrenia: insights from brain imaging studies. European psychiatry: the journal of the Association of European Psychiatrists $2005 ; 20: 15-27$

2. Andreasen NC:Understanding the causes of schizophrenia. The New England journal of medicine 1999; 340:645-647

3. Ayano G: First Generation Antipsychotics: Pharmacokinetics, Pharmacodynamics, Therapeutic Effects and Side Effects: A Review. Research \& Reviews: Journal of Chemistry 2016; 5:53-63

4. Calabrese JR, Keck PE,Jr, Starace A, Lu K, Ruth A, Laszlovszky I, Nemeth G \& Durgam S: Efficacy and safety of low- and high-dose cariprazine in acute and mixed mania associated with bipolar I disorder: a double-blind, placebo-controlled study. The Journal of clinical psychiatry 2015; 76:284-292

5. Caraci F, Leggio GM, Salomone $S$ \& Drago F: New drugs in psychiatry: focus on new pharmacological targets. F1000Research 2017; 6:397

6. Chhatlani A, Farheen SA, Setty MJ \& Tampi RR: Use of cariprazine in psychiatric disorders: A systematic review. Annals of Clinical Psychiatry: Official Journal of the American Academy of Clinical Psychiatrists 2018; 30:326-334

7. Correll CU, Leucht $S \&$ Kane JM: Lower risk for tardive dyskinesia associated with second-generation antipsychotics: a systematic review of 1-year studies. The American Journal of Psychiatry 2004; 161:414-425

8. Durgam S, Cutler AJ, Lu K, Migliore R, Ruth A, Laszlovszky I, Nemeth G \& Meltzer HY: Cariprazine in acute exacerbation of schizophrenia: a fixed-dose, phase 3, randomized, double-blind, placebo- and active-controlled trial. The Journal of clinical psychiatry 2015a; 76:1574

9. Durgam S, Litman RE, Papadakis K, Li D, Nemeth $G$ \& Laszlovszky I: Cariprazine in the treatment of schizophrenia: a proof-of-concept trial. International clinical psychopharmacology 2016a; 31:61-68

10. Durgam S, Starace A, Li D, Migliore R, Ruth A, Nemeth G \& Laszlovszky I: An evaluation of the safety and efficacy of cariprazine in patients with acute exacerbation of schizophrenia: a phase II, randomized clinical trial. Schizophrenia research 2014; 152:450-457

11. Durgam S, Earley W, Guo H, Li D, Nemeth G, Laszlovszky I, Fava $M$ \& Montgomery SA: Efficacy and safety of adjunctive cariprazine in inadequate responders to antidepressants: a randomized, double-blind, placebo-controlled study in adult patients with major depressive disorder. The Journal of clinical psychiatry 2016b; 77:371-378

12. Durgam S, Earley W, Lipschitz A, Guo H, Laszlovszky I, Nemeth G, Vieta E, Calabrese JR \& Yatham LN: An 8Week Randomized, Double-Blind, Placebo-Controlled Evaluation of the Safety and Efficacy of Cariprazine in Patients With Bipolar I Depression. The American Journal of Psychiatry 2016c; 173:271-281

13. Durgam S, Starace A, Li D, Migliore R, Ruth A, Nemeth G \& Laszlovszky I: The efficacy and tolerability of cariprazine in acute mania associated with bipolar I disorder: a phase II trial. Bipolar disorders 2015b; 17:63-75

14. Earley WR, Guo H, Nemeth G, Harsanyi $J$ \& Thase ME: Cariprazine Augmentation to Antidepressant Therapy in Major Depressive Disorder: Results of a Randomized, Double-Blind, Placebo-Controlled Trial. Psychopharmacology bulletin 2018; 48:62-80

15. Earley W, Durgam S, Lu K, Debelle M, Laszlovszky I, Vieta E \& Yatham LN: Tolerability of cariprazine in the treatment of acute bipolar I mania: A pooled post hoc analysis of 3 phase II/III studies. Journal of affective disorders 2017; 215:205-212

16. Egger M, Smith GD and Altman DG: Systematic reviews in health care: meta-analysis in context. 2 nd edn. London: BMJ Books, 2001

17. Fava M, Durgam S, Earley W, Lu K, Hayes R, Laszlovszky $I \&$ Nemeth G: Efficacy of adjunctive low-dose cariprazine in major depressive disorder: a randomized, doubleblind, placebo-controlled trial. International clinical psychopharmacology 2018; 33:312-321

18. Guyatt GH, Oxman AD, Montori V, Vist G, Kunz R, Brozek J, Alonso-Coello P, Djulbegovic B, Atkins D, Falck-Ytter Y, Williams JW,Jr, Meerpohl J, Norris SL, Akl EA \& Schunemann HJ: GRADE guidelines: 5. Rating the quality of evidence--publication bias. Journal of clinical epidemiology 2011; 64: 1277-1282

19. Higgins JP, Altman DG, Gotzsche PC, Juni P, Moher D, Oxman AD, Savovic J, Schulz KF, Weeks L, Sterne JA, Cochrane Bias Methods Group \& Cochrane Statistical Methods Group: The Cochrane Collaboration's tool for assessing risk of bias in randomised trials. BMJ (Clinical research ed.) 2011; 343:d5928

20. Holder SD, Edmunds AL \& Morgan S: Psychotic and Bipolar Disorders: Antipsychotic Drugs. FP essentials 2017; 455:23-29

21. Jablensky A, Sartorius $N$, Ernberg G, Anker M, Korten A, Cooper JE, Day $R$ \& Bertelsen A: Schizophrenia: manifestations, incidence and course in different cultures. A 
World Health Organization ten-country study. Psychological medicine. Monograph supplement 1992; 20:1-97

22. Kane JM, Zukin S, Wang Y, Lu K, Ruth A, Nagy K, Laszlovszky I \& Durgam S: Efficacy and Safety of Cariprazine in Acute Exacerbation of Schizophrenia: Results From an International, Phase III Clinical Trial. Journal of clinical psychopharmacology 2015; 35:367-373

23. Kirkbride JB, Errazuriz A, Croudace TJ, Morgan C, Jackson D, Boydell J, Murray RM \& Jones PB: Incidence of schizophrenia and other psychoses in England, 19502009: a systematic review and meta-analyses. PloS one 2012; 7:e31660

24. Lao KS, He Y, Wong IC, Besag FM \& Chan EW: Tolerability and Safety Profile of Cariprazine in Treating Psychotic Disorders, Bipolar Disorder and Major Depressive Disorder: A Systematic Review with Meta-Analysis of Randomized Controlled Trials. CNS drugs 2016; 30:1043-1054

25. Leucht S, Tardy M, Komossa K, Heres S, Kissling W, Salanti $G$ \& Davis JM: Antipsychotic drugs versus placebo for relapse prevention in schizophrenia: a systematic review and meta-analysis. Lancet (London, England) 2012; 379:2063-2071

26. Nakamura T, Kubota T, Iwakaji A, Imada M, Kapas $M$ \& Morio Y: Clinical pharmacology study of cariprazine (MP214) in patients with schizophrenia (12-week treatment). Drug design, development and therapy 2016; 10:327-338

27. NCT02670538, 2019-last update: Study of the Efficacy of a Fixed-dose Regimen of Cariprazine Compared to Placebo for Treatment of the Depressive Episode in Participants With Bipolar I Disorder. Available: https://www.clinicaltrials.gov/ct2/show/NCT02670538

28. NCT02670551, 2019-last update: Study on the Efficacy, Safety, and Tolerability of Cariprazine Relative to Placebo in Participants With Bipolar I Depression. Available: https://www.clinicaltrials.gov/ct2/show/NCT02670551

29. Ndukwe HC \& Nishtala PS: Glucose monitoring in new users of second-generation antipsychotics in older people. Archives of Gerontology and Geriatrics 2017; 70:136-140

30. NICE, 2014-last update: Psychosis and schizophrenia in adults: prevention and management. Available: https://www.nice.org.uk/guidance/cg178 [06/23, 2017]
31. Rethink Mental Illness, 2015-last update: Schizophrenia. Available: https://www.rethink.org/diagnosis-treatment/ conditions/schizophrenia [06/27, 2017]

32. Robinson D, Woerner MG, Alvir JM, Bilder R, Goldman $R$, Geisler S, Koreen A, Sheitman B, Chakos M, Mayerhoff $D \&$ Lieberman JA: Predictors of relapse following response from a first episode of schizophrenia or schizoaffective disorder. Archives of General Psychiatry 1999; 56:241-247

33. Sachs GS, Greenberg WM, Starace A, Lu K, Ruth A, Laszlovszky I, Nemeth $G$ \& Durgam S: Cariprazine in the treatment of acute mania in bipolar I disorder: a doubleblind, placebo-controlled, phase III trial. Journal of affective disorders 2015; 174:296-302

34. Sapra M, Lawson D, Iranmanesh A \& Varma A: Adiposity-independent hypoadiponectinemia as a potential marker of insulin resistance and inflammation in schizophrenia patients treated with second generation antipsychotics. Schizophrenia research 2016; 174:132-136

35. Schizophrenia Research Institute, 2014-last update: About Schizophrenia. Available:

http://www.schizophreniaresearch.org.au/schizophrenia/a bout-schizophrenia/ [04/17, 2017]

36. Stahl SM: Mechanism of action of cariprazine. CNS spectrums 2016; 21:123-127

37. Steel Z, Marnane C, Iranpour C, Chey T, Jackson JW, Patel $V$ \& Silove D: The global prevalence of common mental disorders: a systematic review and meta-analysis 1980-2013. International journal of epidemiology 2014; 43:476-493

38. Sterne JA, Sutton AJ, Ioannidis JP, Terrin N, Jones DR, Lau J, Carpenter J, Rucker G, Harbord RM, Schmid CH, Tetzlaff J, Deeks JJ, Peters J, Macaskill P, Schwarzer G, Duval S, Altman DG, Moher D \& Higgins JP: Recommendations for examining and interpreting funnel plot asymmetry in meta-analyses of randomised controlled trials. BMJ (Clinical research ed.) 2011; 343: $d 4002$

39. The Nordic Cochrane Centre, The Cochrane Collaboration: Review Manager (RevMan). Version 5.3 edn. Copenhagen: The Nordic Cochrane Centre, 2014

Correspondence:

Ayman Antoun Reyad, PhD

School of Pharmacy, Faculty of Science and Engineering, University of Wolverhampton

Wulfruna Street, Wolverhampton, WV1 1LY, UK

E-mail:a.antounreyad@wlv.ac.uk 